hep-th/0202062

\title{
Wigner Functions for the Landau Problem in Noncommutative Spaces
}

\author{
Ömer F. Dayi ${ }^{a, b}$, and Lara T. Kelleyane ${ }^{a, \text { 巴 }}$ \\ ${ }^{a}$ Physics Department, Faculty of Science and Letters, Istanbul Technical University, \\ 80626 Maslak-Istanbul, Turkey. \\ ${ }^{b}$ Feza Gürsey Institute, P.O. Box 6, 81220, Çengelköy, Istanbul, Turkey.
}

\begin{abstract}
An electron moving on plane in a uniform magnetic field orthogonal to plane is known as the Landau problem. Wigner functions for the Landau problem when the plane is noncommutative are found employing solutions of the Schrödinger equation as well as solving the ordinary $\star$-genvalue equation in terms of an effective Hamiltonian. Then, we let momenta and coordinates of the phase space be noncommutative and introduce a generalized $\star$-genvalue equation. We solve this equation to find the related Wigner functions and show that under an appropriate choice of noncommutativity relations they are independent of noncommutativity parameter.
\end{abstract}

\footnotetext{
${ }^{1}$ E-mail: dayi@itu.edu.tr and dayi@gursey.gov.tr.

${ }^{2}$ E-mail: kelleyane@itu.edu.tr.
} 


\section{Introduction}

Deformation quantization which is also known as Weyl-Wigner-Groenewold-Moyal quantization describes a quantum system in terms of c-number phase space variables [1]. The correspondence with the operator quantization is due to symbol maps and star products. Operators are mapped into c-number functions however, their composition is given by star product which is noncommutative but associative. One of the most important ingredients of deformation quantization is Wigner function[2]. It is essential to define phase space integrals corresponding to expectation values of operator formalism.

When classical coordinates are converted into noncommuting variables they mutate into operators. Thus after performing canonical quantization of noncommutative systems one should deal with two kind of operators. This can be avoided by formulating noncommutativity of coordinates as deformation of commuting ones. One can adopt all the machinery developed in deformation quantization.

An electron moving on plane in a uniform external magnetic field orthogonal to plane is known as the Landau problem. Different aspects of the Landau problem in noncommutative coordinates have been widely studied [3]. Our aim is to derive Wigner functions for the Landau problem for two different noncommuting spaces: When only coordinates are noncommuting and then letting coordinates as well as momenta are noncommuting.

In Section 2 we briefly recall definitions of Wigner function. In Section 3 we study the Landau problem on the noncommutative plane by adopting a differential representation for quantum mechanical operators. This yields Wigner functions which are formal. However, guided by this formulation we define an effective Hamiltonian and obtain Wigner functions utilizing the usual deformation quantization. When both quantum mechanics and noncommutativity are formulated as deformations, it is plausible to consider all of phase space variables on the same footing. Indeed, in Section 4 deformation quantization is adopted by letting coordinates as well as momenta are noncommuting for the Landau problem and the related Wigner functions are calculated. Moreover, we show that noncommutativity relations can be chosen such that the resultant Wigner functions are not aware of noncommutativity of phase space variables. These Wigner functions can be used to calculate some physical quantities in noncommutative spaces. 


\section{Wigner Functions}

Wigner functions can be defined equivalently in two different ways. One of them refers to the

operator formulation of quantum mechanics: Let the Hamiltonian operator be $\hat{\mathcal{H}}$ and define the eigenvalue problem

$$
\hat{\mathcal{H}} \psi(\vec{x})=\mathcal{E} \psi(\vec{x})
$$

If we denote its spectrum by $\mathcal{E}_{l}$ and the corresponding eigenstates by $\psi_{l}(\vec{x})$, (diagonal) Wigner functions are defined as

$$
W_{l}(\vec{x}, \vec{\pi})=\frac{1}{2 \pi} \int d^{d} s \psi_{l}^{*}\left(\vec{x}-\frac{\hbar}{2} \vec{s}\right) e^{-i \vec{s} \cdot \vec{\pi}} \psi_{l}\left(\vec{x}+\frac{\hbar}{2} \vec{s}\right),
$$

where $(\vec{x}, \vec{\pi})$ are $2 d$ dimensional phase space variables.

Wigner functions can also be defined without referring to the operator formalism of quantum mechanics (11), by introducing the $\hbar$-star product

$$
\star_{\hbar} \equiv \exp \frac{i \hbar}{2}\left(\overleftarrow{\partial}_{\vec{x}} \cdot \vec{\partial}_{\vec{\pi}}-\overleftarrow{\partial}_{\vec{\pi}} \cdot \vec{\partial}_{\vec{x}}\right)
$$

Now, the Wigner functions are defined as $\star_{\hbar}$-genvalue equation

$$
\mathcal{H} \star_{\hbar} W_{l}(\vec{x}, \vec{\pi})=\mathcal{E}_{l} W_{l}(\vec{x}, \vec{\pi}),
$$

where $\mathcal{H}$ is the classical Hamiltonian function corresponding to $\hat{\mathcal{H}}$.

These two definitions are equivalent[2]. Wigner function are used to define integrals in the deformation quantization corresponding to expectation values in operator formulation.

\section{Wigner Functions for the Landau Problem in Non- commutative Coordinates}

An electron moving in the plane which is perpendicular to the external uniform magnetic field $B$, neglecting spin, is described by the Hamiltonian

$$
H=\frac{1}{2 m}\left(\vec{p}+\frac{e}{c} \vec{A}\right)^{2} .
$$

where we adopt the symmetric gauge

$$
\vec{A}=\left(-\frac{B}{2} y, \frac{B}{2} x\right) .
$$


Consider the coordinates satisfying

$$
[\tilde{x}, \tilde{y}]=i \theta
$$

where $\theta$ is a constant parameter. Because of being operators even at the classical level, we denoted noncommuting coordinates as $\tilde{x}, \tilde{y}$. Noncommutativity can be imposed by treating the coordinates as commuting, but introducing the $\theta$-star product

$$
\star_{\theta} \equiv \exp \frac{i \theta}{2}\left(\overleftarrow{\partial}_{x} \vec{\partial}_{y}-\overleftarrow{\partial}_{y} \vec{\partial}_{x}\right)
$$

Now, we deal with the commutative coordinates $x$ and $y$ but composition of functions is given by $\theta$-star product (8). For example, instead of the commutator (7) one introduces the Moyal bracket

$$
x \star_{\theta} y-y \star_{\theta} x=i \theta .
$$

We quantize this system in terms of the standard canonical quantization by establishing the usual canonical commutation relations

$$
\left[r_{i}, \hat{p}_{j}\right]=i \hbar \delta_{i j}
$$

and adopting the usual differential representation

$$
\hat{p}_{i}=-i \hbar \frac{\partial}{\delta r_{i}}
$$

Note that we use the same notation for the (quantum) operator and classical coordinates $\vec{r}=(x, y)$.

One of the receipts to define quantum mechanics in noncommutative coordinates is to interpret derivatives as momentum operators: $\vec{\partial}=\frac{i}{\hbar} \hat{\vec{p}}$. According to this receipt, in the symmetric gauge (6) the Hamiltonian operator $\hat{H}$ corresponding to (5), acting on an arbitrary function $\Psi(\vec{r})$ yields

$$
\hat{H} \star_{\theta} \Psi(\vec{r})=\frac{1}{2 m}\left[\left(\hat{p}_{x}-\frac{e B}{2 c} y\right)^{2}+\left(\hat{p}_{y}+\frac{e B}{2 c} x\right)^{2}\right] \star_{\theta} \Psi(\vec{r}) \equiv \hat{H}_{n c} \Psi(\vec{r}),
$$

where, in terms of $\kappa=-\frac{e B \theta}{4 c \hbar}$, we defined

$$
\hat{H}_{n c}=\frac{1}{2 m}\left[\left((1+\kappa) \hat{p}_{x}-\frac{e B}{2 c} y\right)^{2}+\left((1+\kappa) \hat{p}_{y}+\frac{e B}{2 c} x\right)^{2}\right] .
$$


The eigenvalue equation

$$
\hat{H}_{n c} \phi(\vec{r})=E \phi(\vec{r})
$$

can be solved in polar coordinates and its eigenstates can be given in terms of the Laguerre polynomials in $\left(x^{2}+y^{2}\right)$. These solutions are not suitable to find the related Wigner functions although, formally we can write them by making use of the definition (2). Another method may be to use definition of Wigner functions in polar coordinates [4.

We would like to use the fact that effectively the Landau problem is one dimensional harmonic oscillator. The operator

$$
a=\frac{1}{\sqrt{2 m \hbar \tilde{\omega}}}\left[\left(\hat{p}_{x}-\frac{m \tilde{\omega}}{2} y\right)-i\left(\hat{p}_{y}+\frac{m \tilde{\omega}}{2} x\right)\right]
$$

and its hermitian conjugate $a^{\dagger}$ can be shown to satisfy

$$
\left[a, a^{\dagger}\right]=1
$$

where $\tilde{\omega} \equiv(1+\kappa) \omega$ and $\omega=\frac{e B}{m c}$ is the cyclotron frequency.

The Hamiltonian becomes

$$
\hat{H}=\hbar \tilde{\omega}\left(a^{\dagger} a+\frac{1}{2}\right),
$$

which is equivalent to harmonic oscillator in one dimension whose frequency is $\tilde{\omega}$. Thus, its spectrum is given by

$$
E_{n}=\hbar \tilde{\omega}(n+1 / 2),
$$

where $n=0,1, \cdots$. In terms of the ground state

$$
\phi_{0}(\vec{r}, \theta)=\frac{m \tilde{\omega}}{2 \pi \hbar} \exp \left[\frac{-m \tilde{\omega}}{4 \hbar}\left(x^{2}+y^{2}\right)\right]
$$

its eigenfunctions are given as

$$
\phi_{n}(\vec{r}, \theta)=\frac{1}{\sqrt{n !}}\left(a^{\dagger}\right)^{n} \phi_{0} .
$$

These states can be used to write the Wigner functions as

$$
W_{L, n}(\vec{r}, \vec{p}, \theta)=\frac{1}{4 \pi^{2}} \int d^{2} v \phi_{n}^{*}\left(\vec{r}-\frac{\hbar}{2} \vec{v}\right) e^{-i \vec{v} \cdot \vec{p}} \phi_{n}\left(\vec{r}+\frac{\hbar}{2} \vec{v}\right)
$$

However, as it is mentioned above these Wigner functions are only formal, few of the integrals can be calculated under some conditions. 
Although we are using the specific representation (11), let us assume that we can choose the realization

$$
a \psi(q)=\frac{1}{\sqrt{2}}\left(q+\frac{\partial}{\partial q}\right) \psi(q), a^{\dagger} \psi(q)=\frac{1}{\sqrt{2}}\left(q-\frac{\partial}{\partial q}\right) \psi(q),
$$

where the parameter $q$ is a $c$-number. Then, eigenfunctions of (16) coincide with the eigenfunctions of one dimensional harmonic oscillator, which lead to Wigner functions[3]

$$
W_{0, n}(P, q)=\frac{(-1)^{n}}{\pi \hbar} e^{-z} L_{n}(2 z)
$$

where we used the variable

$$
z=\left(P^{2}+q^{2}\right)
$$

$P$ is the parameter appearing in the definition (2).

As we have already mentioned in Section 2, there exists another method of calculating Wigner functions: We can use the $\hbar$-star product (3) and the definition (4). However, in this case we should define a c-number object corresponding to $\hat{H}_{n c}$. Let us define the effective c-number Hamiltonian

$$
H_{e f f}=\frac{(1+\kappa)^{2}}{2 m} \vec{p}^{2}+\frac{m \omega^{2}}{8} \vec{r}^{2}+\frac{\tilde{\omega}}{2}\left(x p_{y}-y p_{x}\right) .
$$

We would like to emphasize the fact that it is not classical because $\kappa$ depends on $\hbar$. Now, to find the related Wigner functions we use the definition

$$
H_{e f f} \star_{\hbar} W_{1}(\vec{p}, \vec{r}, \theta)=E W_{1}(\vec{p}, \vec{r}, \theta)
$$

This can be written as a differential equation only in one variable

$$
\rho \equiv H_{e f f}
$$

as

$$
\rho \frac{d^{2} W_{1}(\rho)}{d \rho^{2}}+\frac{d W_{1}(\rho)}{d \rho}-\frac{4}{\hbar^{2} \tilde{\omega}^{2}}(\rho+E) W_{1}(\rho)=0 .
$$

To solve this equation let us change the variable as

$$
R=\frac{4 \rho}{\hbar \tilde{\omega}}
$$

and write

$$
W_{1}=e^{-R / 2} L(R)
$$


Thus (21) becomes

$$
\left[R \frac{d^{2}}{d R^{2}}+(1-R) \frac{d}{d R}-\left(\frac{1}{2}-\frac{E}{\hbar \tilde{\omega}}\right)\right] W_{1}=0,
$$

whose solutions are Laguerre polynomials $L_{n}(4 R)$ where $n=0,1, \cdots$ and

$$
E_{n}=\hbar \tilde{\omega}(n+1 / 2) .
$$

Thus we have

$$
W_{1, n}(\vec{p}, \vec{r}, \theta)=e^{-2 H_{e f f} / \hbar \tilde{\omega}} L_{n}\left(4 H_{e f f} / \hbar \tilde{\omega}\right) .
$$

Indeed, this is not surprising: The system is equivalent to one dimensional harmonic oscillator, which also can be observed at the "classical level". By performing the canonical transformation

$$
\begin{aligned}
& p_{1}=\frac{(1+\kappa)}{\sqrt{m}} p_{x}-\frac{\omega \sqrt{m}}{2} y, q_{1}=\frac{(1+\kappa)}{\sqrt{m}} p_{y}+\frac{\omega \sqrt{m}}{2} x, \\
& p_{2}=\frac{(1+\kappa)}{\sqrt{m}} p_{y}-\frac{\omega \sqrt{m}}{2} x, q_{2}=\frac{(1+\kappa)}{\sqrt{m}} p_{x}+\frac{\omega \sqrt{m}}{2} y,
\end{aligned}
$$

the effective Hamiltonian becomes

$$
H_{\text {eff }}=\frac{1}{2}\left(p_{1}^{2}+q_{1}^{2}\right)
$$

The other set of variables $\left(p_{2}, q_{2}\right)$ leads to the degeneracy of the Landau levels after quantization.

As it may be expected, using the effective Hamiltonian (19) and assuming the representation (17) yield the same Wigner functions.

\section{Wigner Functions for the Landau Problem in Non- commutative Phase Space}

Quantum mechanics in noncommutative space can be defined in terms of Wigner functions found by making use of the $\star_{\hbar}$ and $\star_{\theta}$ simultaneously. However, when $\star_{\hbar}$-genvalue equations are considered it is plausible to treat $\vec{r}$ and $\vec{p}$ on the same footing. Thus, we would like to deform also momenta of the phase space by introducing

$$
\star_{k} \equiv \exp \frac{i k \theta}{2}\left(\overleftarrow{\check{\partial}}_{p_{x}} \vec{\partial}_{p_{y}}-\overleftarrow{\partial}_{p_{y}} \vec{\partial}_{p_{x}}\right)
$$

where $k$ is any constant. 
Now, consider

$$
\star \equiv \star_{\hbar} \star_{\theta} \star_{\theta^{ \pm}}
$$

which we use to define the $\star$-genvalue equation

$$
H \star \Psi=E \Psi,
$$

where $H$ is the classical Hamiltonian (5) of the Landau problem in the symmetric gauge (66).

A similar approach for harmonic oscillator was given in [5].

*-genvalue equation can be written as

$$
\begin{gathered}
\left\{H-\frac{\hbar^{2}}{8}\left[\frac{1}{m} \vec{\partial}_{r} \cdot \vec{\partial}_{r}+\frac{m \omega^{2}}{4} \vec{\partial}_{p} \cdot \vec{\partial}_{p}-\omega\left(\partial_{p_{x}} \partial_{y}-\partial_{p_{y}} \partial_{x}\right)\right]\right. \\
-\frac{\theta^{2}}{8}\left[\frac{m \omega^{2}}{4} \vec{\partial}_{r} \cdot \vec{\partial}_{r}+\frac{k^{2}}{m} \vec{\partial}_{p} \cdot \vec{\partial}_{p}+k \omega\left(\partial_{p_{x}} \partial_{y}-\partial_{p_{y}} \partial_{x}\right]\right. \\
+\frac{\theta \hbar}{4}\left[\frac{\omega}{2} \vec{\partial}_{r} \cdot \vec{\partial}_{r}+k \vec{\partial}_{p} \cdot \vec{\partial}_{p}+\left(\frac{\omega^{2} m}{4}+\frac{k}{m}\right)\left(\partial_{x} \partial_{p_{y}}-\partial_{y} \partial_{p_{x}}\right)\right. \\
-\frac{i \hbar}{2}\left[\frac{1}{m} \vec{p} \cdot \vec{\partial}_{r}-\frac{m \omega^{2}}{4} \vec{r} \cdot \vec{\partial}_{p}-\frac{\omega}{2}\left(y_{x} \partial_{x}-p_{x} \partial_{p_{y}}-x \partial_{y}+p_{y} \partial_{p_{x}}\right)\right] \\
\left.+\frac{i \theta}{2}\left[\frac{m \omega^{2}}{4}\left(x \partial_{y}-y \partial_{x}\right)+\frac{k}{m}\left(p_{x} \partial_{p_{y}}-p_{y} \partial_{p_{x}}\right)+\frac{\omega}{2}\left(p_{y} \partial_{y}+y \partial_{p_{y}}-k x \partial_{p_{x}}-k p_{x} \partial_{x}\right)\right]-E\right\} W_{2}=0
\end{gathered}
$$

where $\vec{\partial}_{r} \equiv \partial / \partial \vec{r}$ and $\vec{\partial}_{p} \equiv \partial / \partial \vec{p}$. Introducing the variable

$$
\zeta \equiv H
$$

one can show that the imaginary part of (31) vanishes and it reads

$$
-4 \alpha^{2}(k, \theta, \hbar)\left(\zeta \frac{d^{2} W_{2}(\zeta)}{d \zeta^{2}}+\frac{d W_{2}(\zeta)}{d \zeta}\right)+\zeta W_{2}(\zeta)=E W_{2}(\zeta),
$$

where we defined

$$
\alpha^{2}(k, \theta, \hbar)=\frac{1}{16} \hbar^{2} \omega^{2}+\frac{\theta^{2}}{16}\left(\frac{k^{2}}{m^{2}}+\frac{\omega^{4} m^{2}}{16}+\frac{k \omega^{2}}{2}\right)-\frac{1}{2} \hbar \theta\left(\frac{\omega^{3} m}{4}+\frac{k \omega}{m}\right) .
$$

In terms of the variable

$$
\eta=\frac{\zeta}{\alpha(k, \theta, \hbar)}
$$

(33) can be written as

$$
\left(\eta \partial_{\eta}{ }^{2}+\partial_{\eta}-\eta / 4+\tilde{E}\right) W_{2}(\eta)=0,
$$

where $\tilde{E}=\frac{E}{4 \alpha(k, \theta, \hbar)}$. By writing

$$
W_{2}(\eta)=e^{-\eta / 2} L(\eta)
$$


we see that $L(\eta)$ satisfies the differential equation

$$
\left[\eta \partial_{\eta}^{2}+(1-\eta) \partial_{\eta}+\tilde{E}-1 / 2\right] L(\eta)=0 .
$$

Thus $L(\eta)$ is given as Laguerre polynomials $L(\eta)=L_{n}(4 \eta)$ and

$$
E_{k, n}=4 \alpha(k, \theta, \hbar)(n+1 / 2),
$$

where as usual $n=0,1, \cdots$. Therefore, Wigner functions of the Landau problem in noncommutative phase space are

$$
W_{2, n}(\vec{p}, \vec{r}, k, \theta)=\frac{(-1)^{n}}{\pi \hbar} e^{-H / 2 \alpha(k, \theta, \hbar)} L_{n}(4 H / \alpha(k, \theta, \hbar)) .
$$

There exists a very interesting case: When

$$
k \equiv k_{s}=-\frac{1}{4} \omega^{2} m^{2}
$$

$\alpha(k, \theta, \hbar)$ becomes independent of $\theta$ :

$$
\alpha^{2}\left(k_{s}, \theta, \hbar\right)=\frac{\hbar^{2} \omega^{2}}{16}
$$

Thus, noncommutativity relations of phase space can be taken such that the resultant quantum mechanics is not aware of noncommutativity.

One can use the Wigner functions (35) to calculate physical quantities. One of the first applications is to calculate the quantities considered in other frameworks [3] and compare the results. Nevertheless, this approach constitutes an alternative method of defining the Landau problem in noncommutative classical phase space. 


\section{References}

[1] H. Weyl, Z. Phys. 46 (1927) 1;

E. Wigner. Phys. Rev. 40 (1932) 749;

H.J. Groenewold, Physica 12 (1946) 405;

J. Moyal, Proc. Cambridge Philos. Soc. 45 (1949) 99.

[2] M. Hillery, R.F. O'Connell, M.O. Scully and E.P. Wigner, Phys. Rep. 106 (1984) 121;

T. Curtright, D. Fairlie and C. Zachos, Phys. Rev. D 58 (1998) 025002;

M. Levanda and V. Fleurov, Ann. Phys. 292 (2001) 199;

C. Zachos, Deformation Quantization: Quantum Mechanics Lives and Works in PhaseSpace, hep-th/0110114.

[3] L. Mezincescu, "Star Operation in Quantum Mechanics" hep-th/0007046;

V.P. Nair and A.P. Polychronakos, Phys. Lett. B 505 (2001) 267, hep-th/0011172;

J. Gamboa, F. Mendez, M. Loewe and J.C. Rojas, Mod. Phys. Lett. A 16 (2001) 2075, hep-th/0109186 and hep-th/0104224;

D.H. Correa, G.S. Lozano, E.F. Moreno and F.A. Schaposnik, Mod. Phys. Lett. A 16 (2001) 2075, hep-th/0109186;

(for a review of earlier work) R. Jackiw, "Physical Instances of Noncommuting Coordinates", hep-th/0110057;

Ö.F. Dayi and A. Jellal, Phys. Lett. A 287 (2001) 349, cond-mat/0103562;

A. Jellal, J. Phys. A 34 (2001) 10159, hep-th/0105303;

Ö.F. Dayi and A. Jellal, Hall Effect in Noncommutative Coordinates, hep-th/0111267.

[4] J. Twamley, J. Phys. A 31 (1998) 4811;

T. Hakioğlu, J. Opt. Soc. Am. A 17 (2000) 2411.

[5] A. Hatzinikitas and I. Smyrnakis, J. Math. Phys. 43 (2002) 113. 